\title{
TOPOGRAFÍA SUPERFICIAL Y SUBGLACIAR DE UN SECTOR ALEDAÑO A LA BASE O'HIGGINS, UBICADA EN EL EXTREMO NORTE DE LA PENÍNSULA ANTÁRTICA
}

\author{
SURFACE AND SUBGLACIER TOPOGRAPHY NEAR TO THE O'HIGGINS \\ BASE STATION AT NORTH OF THE ANTARCTIC PENINSULA
}

Carlos Cárdenas M. ${ }^{1,2}$, Erling Johnson G. ${ }^{1}$, Rubén Carvallo B. ${ }^{1,3}$

\begin{abstract}
In January 2009, Radio Echo Sounding (RES) measures were made during the campaign in the northern part of the Antarctic Peninsula, at the surroundings of the Chilean Base Bernardo O'Higgins (63 $\left.19^{\prime} \mathrm{S} ; 57^{\circ} 53^{\prime} \mathrm{W}\right)$. The system has three main components: Transmitter, Receiver and a Data Acquisition System. The transmitting antenna, penetrating the ice and then returning to the receptor after their reflection in an internal target or bedrock, carries out radio frequency signals generated by the transmitter. The records are stored in a data acquisition system for post processing purposes. All radar data collected was georeferenced with post-processed GPS measurements. With the Radar and GPS data we will obtain the surface and subglacial topography.
\end{abstract}

Key words: radar, surface and subglacial topography.

\section{RESUMEN}

En enero de 2009, durante una campaña al sector norte de la Península Antártica específicamente en los alrededores de la Base Chilena Bernardo O'Higgins, ubicada en el islote Isabel Riquelme de la rada Covadonga en el cabo Legoupi (63 $19^{\prime} \mathrm{S}$; 57 $53^{\circ} \mathrm{W}$ ), se realizaron mediciones de espesor del hielo utilizando un sistema de radar de impulso que consta de tres componentes principales: Transmisor, Receptor y un Sistema de Adquisición de Datos. El transmisor genera una señal de radio frecuencia que es transmistida hacia el interior del hielo mediante una antena transmisora para luego capturar la señál de retorno de la estratigrafía interna del hielo o del fondo rocoso para posteriormente almacenar la información en el sistema de adquisición de datos donde mediante un análisis de retardo de la señal se

1 Dirección de Programas Antárticos, Universidad de Magallanes, Casilla 113-D, Punta Arenas, Chile.

2 Centro de Estudios del Cuaternario, CEQUA, Avenida Bulnes 01855, Casilla 113-D, Punta Arenas, Chile.

3 Departamento de Ingeniería Eléctrica, Universidad de Magallanes, Casilla 113-D, Punta Arenas, Chile. 
determina la distancia entre la antena y cada estrato como asi también el fondo subglaciar. Con el objetivo de establecer la posición geográfica de cada medición de profundidad en forma complementaria se geo-referenció mediante equipos GPS de calidad topografíca. Se señalarán los procesos de análisis de los datos y presentarán los resultados, obteniendo la topografía superficial y subglacial de la zona estudiada.

Palabras clave: radar, topografía superficial y subglacial.

\section{INTRODUCCIÓN}

Las actividades de terreno fueron desarrolladas sobre la Meseta Infantería $\left(63^{\circ} 19^{\prime} 49^{\prime \prime} \mathrm{S}, 57^{\circ}\right.$ 52 ' 28" O) (Fig. 1), ubicada en el extremo norte del sector antártico peninsular en la costa del estrecho de Bransfield. La figura 1 muestra la grilla trazada y los recorridos realizados con el sistema de radar de superficie sobre el sector donde normalmente anevizan aviones Twin Otter. El sistema de radar utilizado para realizar las mediciones sobre la superficie glaciar se grafica en la figura 2. Este sistema consiste de un mototobogan y dos trineos de arrastre sobre los cuales se transportan el transmisor, el receptor y el sistema de adquisición de datos junto a sus respectivas antenas. Además se presenta un radargrama con parte del trayecto realizado sobre la Meseta Infantería, equivalente a una sección de la grilla trazada sobre la zona que se utiliza como pista de anevizaje. Con el objetivo de establecer la dinámica del glaciar se instalaron balizas en la superficie, las que permitirán obtener, mediante la remedición en un periodo deteminado, la velocidad y dirección del hielo. La totalidad de las mediciones realizadas en terreno fueron georeferenciadas utilizando un sistema de posicionamiento global (GPS) de calidad topográfica.

\section{MATERIALES Y MÉTODOS}

El trabajo realizado en terreno contempla la adquisición de imágenes de radar, mediante un equipo transmisor de impulso con una frecuencia central de $5 \mathrm{Mhz}$; el receptor se encuentra compuesto por un osciloscopio, un balun y antenas del tipo dipolo resistivo, de similares características a las instaladas en el transmisor, en complemento con un sistema de adquisición de datos (Fig. 2).

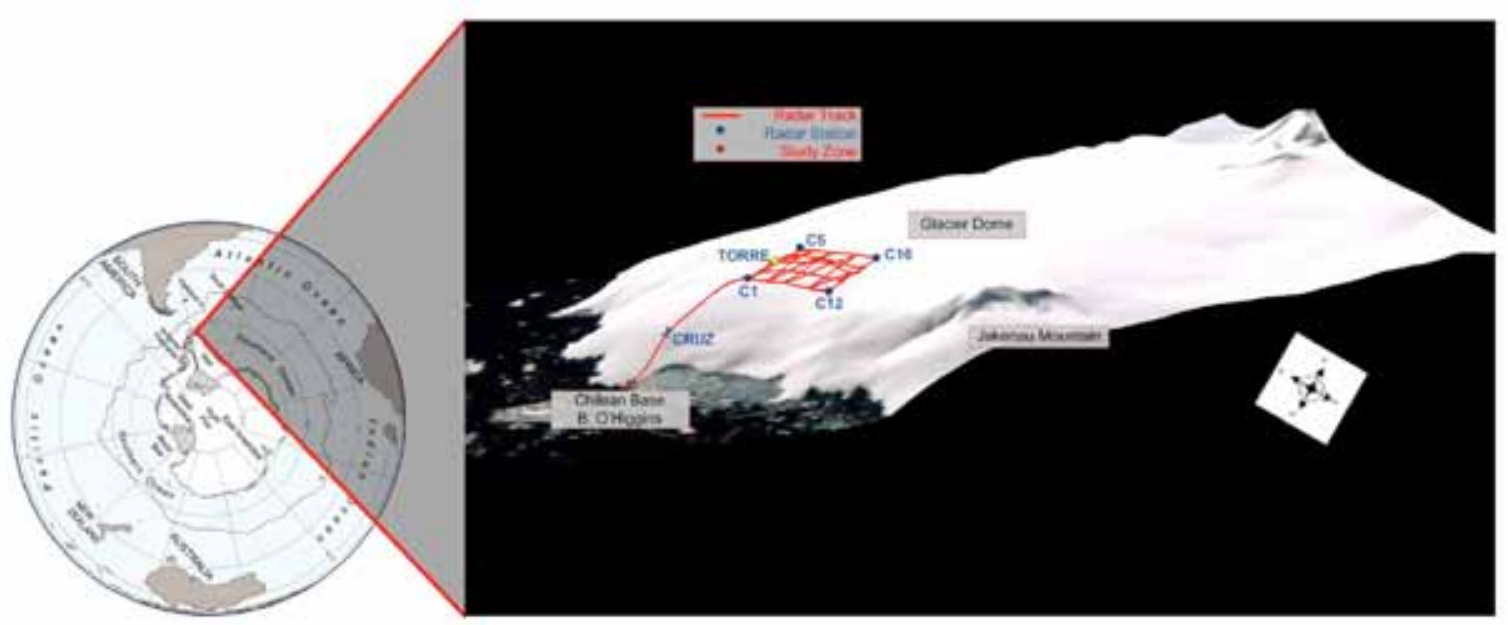

Fig. 1. Sector Base Chilena Bernardo O'Higgins ubicada en el islote Isabel Riquelme de la rada Covadonga en el cabo Legoupi. (Modelo digital de terreno GDEM, sobre imagen Landsat ${ }^{4}$ ). 


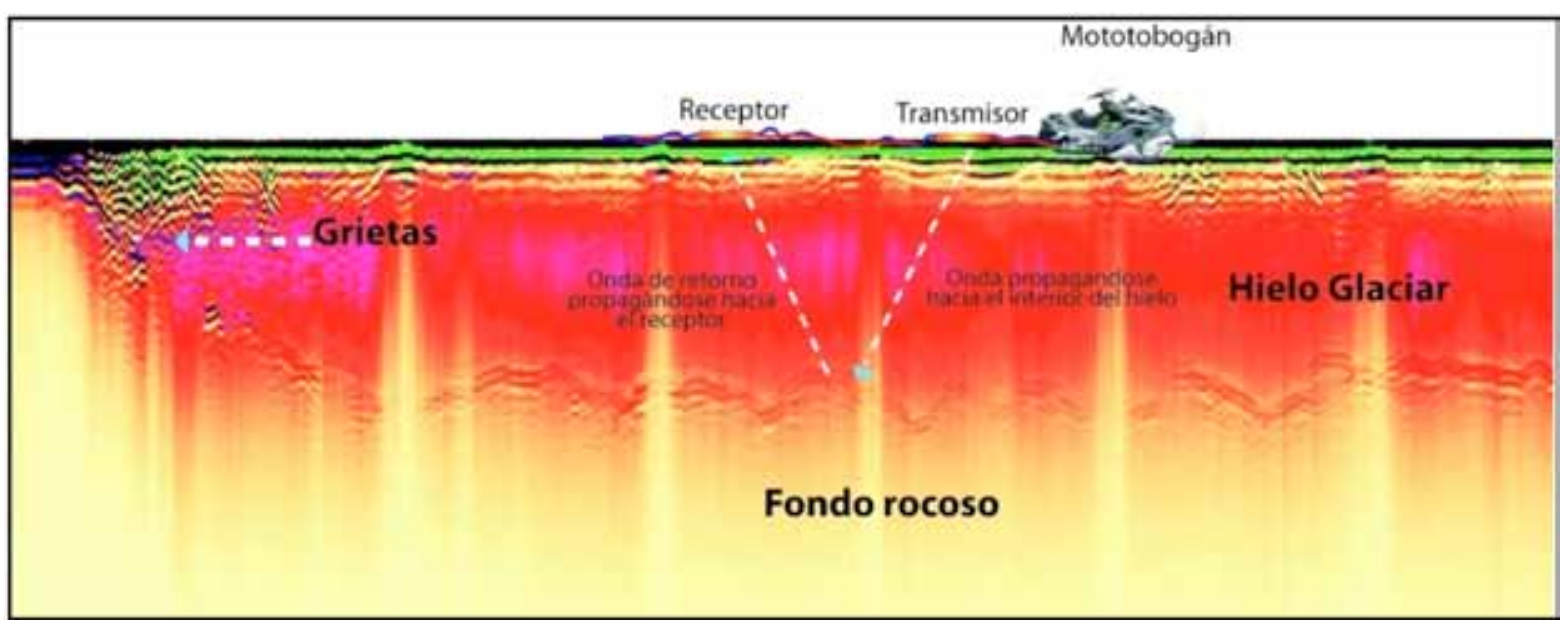

Fig. 2. Configuración del sistema de Radar utilizado.

El sistema de radar de impulso se basa en la emisión de ondas de radio frecuencias. Un pulso de alta energía (cercano a $1600 \mathrm{~V}_{\text {peak-peak }}$ ) pero de corta duración (alrededor de 100 ns) se emite a través de la antena transmisora propagándose en parte por el aire y otra por el hielo, cuando esta última atraviesa la frontera entre dos medios con diferente constante dieléctrica parte de la señal retorna a la superficie mientras que otra se propaga por el nuevo medio cambiando su velocidad de acuerdo a los parámetros eléctricos del material para finalmente alcanzar la antena receptora donde la señal de retorno es almacenada en el sistema de adquisición de datos.

Los datos obtenidos mediante el sistema de radar son georeferenciados mediante un sistema GPS topográfico compuesto por dos equipos del tipo "smart-antenna" marca Novatel, uno de ellos utilizado como estación base mientras que el otro es utilizado como móvil. Esta configuración permite identificar los puntos geográficos donde se realizaron las mediciones de Radar con una mayor presición. Después del terreno los datos se procesan utilizando el software Trimble Pathfinder.

Los datos radar son digitalizados mediante un osciloscopio y un computador del tipo portátil (handheld) los captura a través de un software especialmente diseñado para este proceso, adquiriendo la señal según una configuración pre-establecida de número de trazas y cantidad de valores promediados. Estos datos de radar se muestran en una imagen de radar o radargrama utilizando el software IDRISI ${ }^{\circledR}$, el cual despliega la imagen en pantalla (Fig. 3), per-

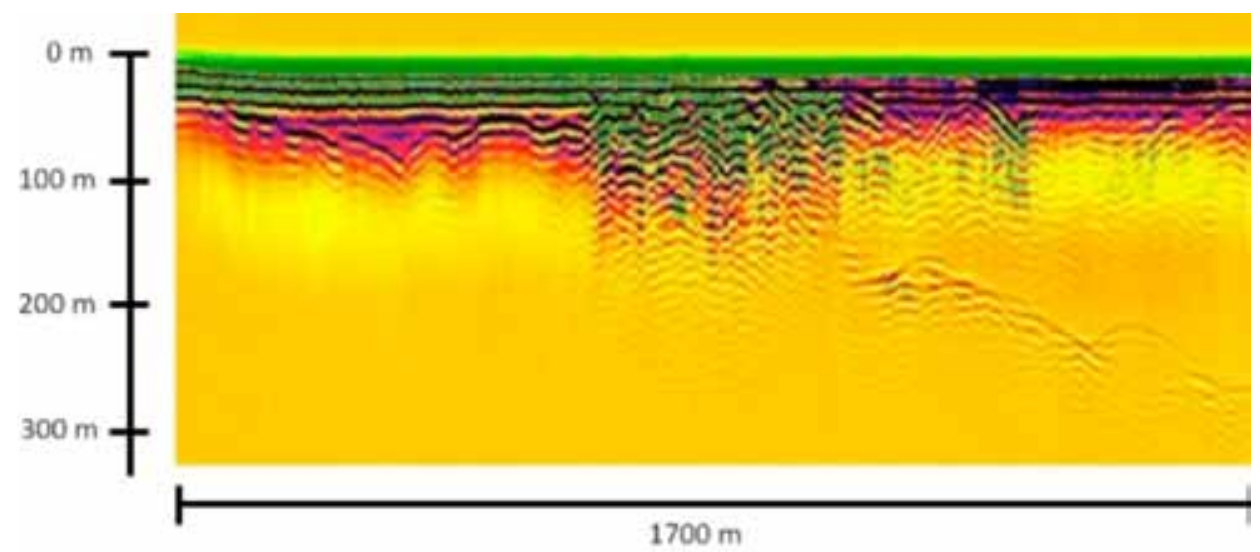

Fig. 3. Radargrama donde se observa el retorno de la topografia sub-glaciar. 
Tabla 1. Espesor (E; m) para cada punto GP.

\begin{tabular}{lcccccccccccccccc}
\hline GPS & C1 & C2 & C3 & C4 & C5 & C6 & C7 & C8 & C9 & C10 & C11 & C12 & C13 & C14 & C15 & C16 \\
E & 255 & 286 & 279 & 262 & 307 & 255 & 281 & 262 & 287 & 267 & 302 & 255 & 287 & 282 & 274 & 265 \\
\hline
\end{tabular}

mitiendo posteriormente digitalizar el fondo rocoso del glaciar y exportar los valores a un vector de datos desde donde son fácilmente reconocibles por cualquier programa de análisis numérico como por ejemplo EXCEL ${ }^{\circledR}$ o MATLAB ${ }^{\circledR}$. Cuando la información se encuentra en forma de vector, es posible obtener el espesor del hielo mediante las ecuaciones que se expondrán a continuación.

\section{CÁLCULO DEL ESPESOR}

La relación que interpreta el espesor del hielo (Watts and Isherwood, 1978) se encuentra definida por:

$$
D=\sqrt{t+\frac{s^{2}}{c} \frac{c^{2}}{4 \varepsilon_{i}}-\frac{s^{2}}{4}}
$$

donde:

$\mathrm{D}=$ Espesor del hielo $(\mathrm{m})$

$\mathrm{S}=$ Distancia entre el transmisor y el receptor (m)

$\mathrm{C}=$ Velocidad de la señal en el vacío $(\mathrm{m} / \mathrm{s})$

$\varepsilon_{\mathrm{I}}=$ Permitividad dieléctrica

El tiempo, por otro lado, corresponde a:

$$
t=\frac{t_{\text {escala }}}{n_{\text {puotos }}} n_{\text {divisiomes }}\left(P_{\text {eco }}-P_{\text {disparo }}\right)
$$

donde:

$$
\begin{array}{ll}
\mathrm{t}_{\text {escala }} & =\text { Escala de tiempo }(\mathrm{s}) \\
\mathrm{n}_{\text {puntos }} & =\text { Número de puntos de la pantalla } \\
\mathrm{n}_{\text {divisiones }} & =\text { Número de divisiones de la pantalla }
\end{array}
$$

$$
\begin{array}{ll}
\mathrm{P}_{\text {eco }} & =\text { Pixel de inicio del eco } \\
\mathrm{P}_{\text {disparo }} & =\text { Pixel de inicio del disparo }
\end{array}
$$

Los datos necesarios para evaluar cada ecuación se encuentran presentes por un lado en el encabezado de cada pulso de radar obtenido por el osciloscopio y posteriormente en el análisis de cada pixel en el "raster".

\section{RESULTADOS}

Recopilando los datos digitalizados es posible resolver las ecuaciones (2.1) y (2.2), obteniendo los espesores en metros para cada punto GPS (Tabla 1).

A continuación se procede a geo-referenciar cada punto GPS con los datos obtenidos gracias al post-proceso diferencial. Los resultados de cada punto (Fig. 4) con su coordenada y elevación sobre el nivel del mar se presentan en la Tabla 2, además se calculó la profundidad de la base glaciar.

Como muestra la figura 4 los valores de espesor del glaciar varian entre un mínimo de $200 \mathrm{~m}$ a un máximo de $260 \mathrm{~m}$ desde la superficie a la base. Los resultados muestran que un sector del fondo rocoso se encuentra bajo el nivel del mar. Esto a partir del cálculo de la diferencia entre el espesor de hielo, obtenida mediante el sistema de radar y la elevación de la superficie glaciar resultante entregada por el post-procesamiento diferencial del GPS. El vector correspondiente a la topografía subglacial fue obtenida a través de la digitalización de cada uno de los radargramas, mientras que la topografía superficial

\begin{tabular}{|c|c|c|c|c|c|c|c|c|c|c|c|c|c|c|c|c|}
\hline GPS & C1 & $\mathrm{C} 2$ & C3 & $\mathrm{C} 4$ & C5 & C6 & C7 & $\mathrm{C} 8$ & C9 & C10 & C11 & C12 & C13 & C14 & C15 & C16 \\
\hline$E$ & 255 & 286 & 279 & 262 & 307 & 255 & 281 & 262 & 287 & 267 & 302 & 255 & 287 & 282 & 274 & 265 \\
\hline A & 207 & 210 & 209 & 211 & 216 & 214 & 226 & 218 & 235 & 221 & 248 & 219 & 231 & 242 & 252 & 259 \\
\hline Prof & -48 & -76 & -70 & -51 & -91 & -41 & -55 & -44 & -52 & -46 & -54 & -37 & -56 & -40 & -22 & -6 \\
\hline
\end{tabular}
se generó a través de los puntos obtenidos por el GPS (de acuerdo al orden el cual fueron capturados desde: $\mathrm{C} 2$ a C7) y los puntos intermedios obtenidos mediante el software Surfer $($, utilizando el método "kriging".

Tabla 2. Puntos GPS, espesor de la base glaciar (E; m), altitud (A; msnm) y profundidad (Prof; msnm). 


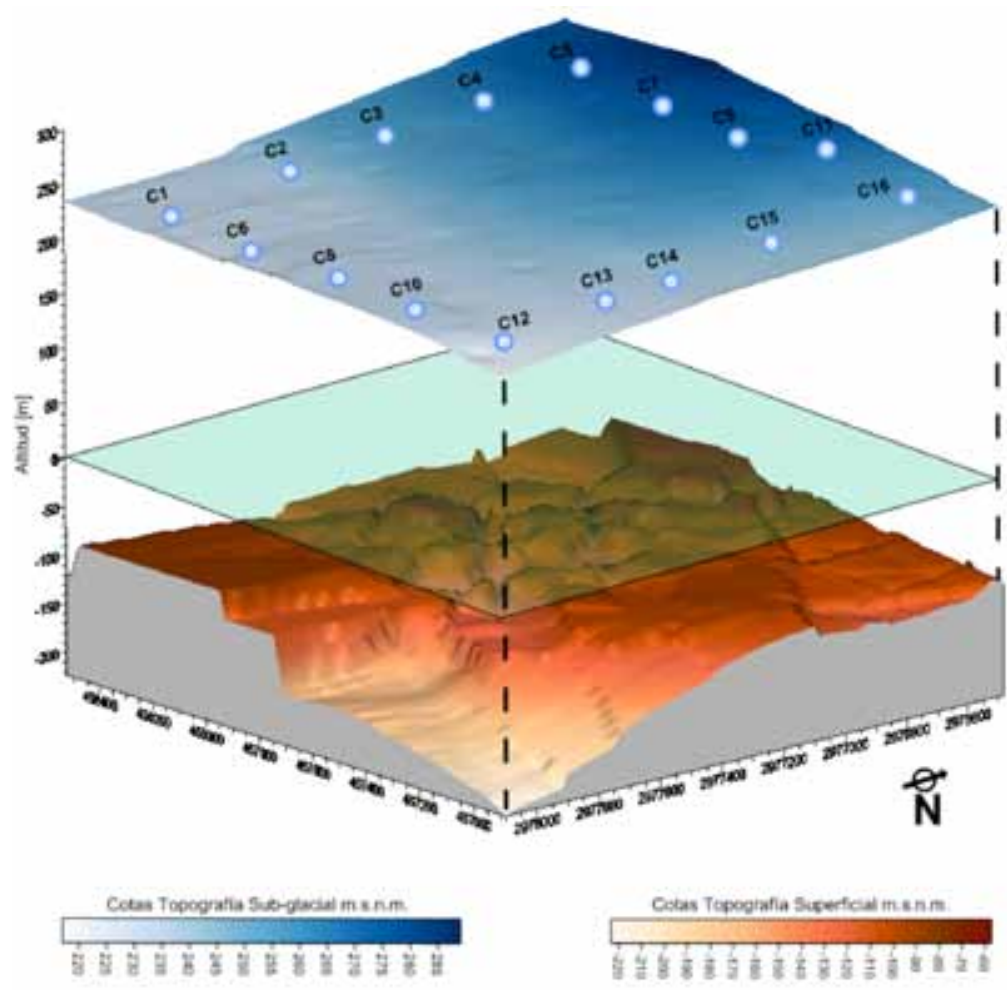

Fig. 4. Diagrama que representa la topografía sublacial y superficial, indicandose la cota cero correspondiente al nivel del mar.

\section{CONCLUSIONES}

a) La utilización de un sistema de radar transportado en forma superficial sobre el glaciar (denominado domo de la Infantería) por medio de un Skydoo y dos trineos permitió capturar información en forma continua relacionada con la caracterización física del glaciar.

b) El haber sondeado sobre la grilla descrita en la fig. 1 permitió aumentar la información de la topografía superficial y subglacial del sector que normalmente es utilizada como pista de anevizaje por aviones del tipo Twin Otter.

c) Sobre el sector utilizado como pista de anevizaje no se detectaron caracteristicas físicas que puedan ser atribuibles a la presencia de grietas.

d) Los equipos GPS permitieron obtener la topografia superficial con un error menor a $16 \mathrm{~m}$ (según el fabricante), para luego de un post proceso diferencial presentar una precisión centimétrica.

e) Los registros de radar de superficie permitieron obtener satisfactoriamente la topografía subglacial del sector en estudio, donde se muestra que parte del fondo rocoso del glaciar se encuentra bajo el nivel del mar.

\section{AGRADECIMIENTOS}

Al Departamento Antártico del Ejército de Chile (DAE) por su apoyo logístico a la Dotación Antártica 2009 de la base O'Higgins y los miembros de la Brigada de Reparaciones, por el apoyo logístico proporcionado al desarrollo del proyecto. Estas actividades son financiadas bajo el alero del proyecto interno UMAG, N²7501 (PR-F1-DPA-2009).

\section{LITERATURA CITADA}

Watts, R.D. \& W. Isherwood 1978. Gravity surveys in glacier-covered regions. Geophysics, (4):819-822

Casassa, G. 1992. Radio-echo sounding of Tyndall Glacier, southern Patagonia. Bulletin of Glacier Research 10 (1992) 60-74 Date Center for Glaciar Research, Japanese Society of Snow and Ice 
